\title{
Philippe Gasparini, Poétiques du je. Du roman autobiographique à l'autofiction
}

\section{Francesca Forcolin}

\section{Q OpenEdition}

1 Journals

\section{Edizione digitale}

URL: http://journals.openedition.org/studifrancesi/5479

DOI: 10.4000/studifrancesi.5479

ISSN: 2421-5856

\section{Editore}

Rosenberg \& Sellier

\section{Edizione cartacea}

Data di pubblicazione: 1 dicembre 2016

Paginazione: 585-586

ISSN: 0039-2944

\section{Notizia bibliografica digitale}

Francesca Forcolin, «Philippe Gasparini, Poétiques du je. Du roman autobiographique à l'autofiction »,

Studi Francesi [Online], 180 (LX | III) | 2016, online dal 01 janvier 2017, consultato il 18 septembre 2020. URL : http://journals.openedition.org/studifrancesi/5479; DOI : https://doi.org/10.4000/studifrancesi. 5479

Questo documento è stato generato automaticamente il 18 settembre 2020.

\section{(c)}

Studi Francesi è distribuita con Licenza Creative Commons Attribuzione - Non commerciale - Non opere derivate 4.0 Internazionale. 


\title{
Philippe Gasparini, Poétiques du je. Du roman autobiographique à l'autofiction
}

\author{
Francesca Forcolin
}

\section{NOTIZIA}

PHILIPPE GASPARINI, Poétiques du je. Du roman autobiographique à l'autofiction, Lyon, Presses

Universitaires de Lyon, 2016, $270 \mathrm{pp}$.

1 Questo volume fa parte della collana «A(etc.)» diretta da Roger-Yves Roche, dove la lettera dell'alfabeto sta per «Autofictions»: l'interesse parte già dal titolo, la designazione al plurale mira a mostrare la molteplicità e vivacità di un genere letterario mai statico, la cui definizione, dopo quasi quarant'anni dalla nascita del termine ad opera di Serge Doubrovsky, continua tuttora a mutare. Autofiction è «mille manières de jouer avec le je», mille modi di esporsi nascondendosi tra le righe di un libro, tra il bianco e il nero di una fotografia, aprendosi alle svariate forme d'arte, come sottolinea la presentazione della collana in prima pagina. In questo numero di «A(etc.)», Philippe Gasparini, specialista della scrittura in prima persona e autore, tra $\mathrm{i}$ tanti, di un importante volume edito nel 2004 presso Seuil, Est-il je?, prosegue e approfondisce gli studi sullo spazio autobiografico e le sue molteplici ambiguità, raccogliendo articoli e atti di conferenze - alcuni già precedentemente pubblicati in rivista - in cui esplora l'evoluzione dell'io in testi chiave dall'ottocento a oggi. L'obiettivo è quello di definirne la poetica (da qui il titolo del volume), ricostruire gli strumenti adottati dallo scrittore in testi che richiedono un lavoro di vera e propria investigazione da parte del lettore, alla ricerca di indizi per svelare il significato, o meglio i significati e le possibili interpretazioni intrinseche nell'opera.

2 Gli undici articoli possono essere suddivisi, per comodità del lettore, in alcune sezioni. La prima comprende gli studi consacrati a René, L'Insurgé e Portrait de l'artiste en jeune 
homme: Gasparini non si limita a proporre un'analisi strutturale, ma mette in evidenza tutte quelle caratteristiche che fanno di questi testi dei possibili precursori dell' autofiction, anche se l'interesse di questa etichetta, a suo parere, sta nel rendere conto di un fenomeno strettamente contemporaneo, il ritorno cioè del soggetto e dell'autore, sintomatico dell'individualismo e del narcisismo "postmoderni". René (Chateaubriand: naissance d'un genre, pp.11-26), mediante indizi onomastici, commenti intertestuali e una narrazione retrospettiva che attestano una volontà autobiografica, apre la strada a un genere nuovo, un "coming out fictionnalisé» da cui prende ispirazione la generazione di Doubrovsky, Roth e Ôé. Vallès, invece, con Vingtras, protagonista di L'Insurgé (Jules Vallès: stratégie de l'insurgé, pp. 27-68), rompe con l'eroe romantico per costruire la figura di un rivoltoso attraverso una lingua orale che anticipa il monologo interiore - oralità che è, secondo la definizione di Gasparini, uno dei caratteri distintivi dell'autofiction. Molteplici sono, in L'Insurgé, i riferimenti autobiografici, dalla sonorità dei nomi (Jacques Vingtras ha un ritmo fonetico simile a Jules Vallès) al contesto storico del racconto che mescola sfera individuale e collettiva, alla biografia dell'autore posta alla fine del volume, all'intertesto con le autocitazioni. Joyce (James Joyce: mythologie de l'artiste, pp. 69-96) è la dimostrazione che questi procedimenti letterari non sono propri dell'ambito francese: una serie di elementi paratestuali nel racconto eterodiegetico del Portrait de l'artiste en jeune homme danno informazioni sui punti in comune tra il protagonista Dedalus e il suo autore. Le opere dei due premi Nobel Kertész e Xingjian (Imre Kertész, Gao Xingjian: deux romans-témoignages, pp. 97-130) sono simili per obiettivo: gli autori, confrontati con le esperienze limite della deportazione e della persecuzione, hanno cercato un dispositivo per rendere la loro esperienza comunicabile. Kertész, in Être sans destin, ha dato alla sua testimonianza una forma romanzesca, fingendo di narrare la realtà della Shoah in simultanea con i fatti accaduti, senza adottare pertanto un punto di vista retrospettivo; Xingjian, invece, in Livre d'un homme seul rifiuta la prima persona narrativa, utilizzando la posizione di un narratore obiettivo ed esterno ai fatti per dare al suo romanzo lo statuto della testimonianza. Di natura differente è invece l'approccio alla scrittura di Annie Ernaux, in particolare in due opere pubblicate a dieci anni di distanza e che trattano il medesimo rapporto tra il je e l'amante: Annie Ernaux: de "Se perdre" à "Passion Simple" (pp. 131-150). Il primo è un diario, il secondo il racconto dell'esperienza vissuta. La differenza (e l'interesse) risiede, ci dice l'A., nel rapporto di tali testi con la temporalità: l'autrice, sottomettendo i propri ricordi a procedure di frammentazione e analisi, consegna al lettore due concetti differenti di verità letteraria, attraverso una temporalità sospesa in Passion Simple e ossessiva nel diario. Dove si trova dunque il grado più alto di sincerità: nel getto spontaneo diaristico o nel testo rielaborato in vista di pubblicazione? Nell'articolo successivo, Gombrowicz: limites de l'autofabulation (pp.151-174), Gasparini riprende il concetto di «autofabulation», inteso come una strategia narrativa che permette al soggetto, il cui nome coincide con quello dell'autore, di inventare un'esistenza immaginaria, saturando così il racconto di elementi inverosimili. È il caso di Ferdydurke di Gombrowicz, romanzo ricco di indizi autobiografici ma in cui la finzione lo spoglia da ogni funzione referenziale.

3 I due articoli successivi, Autofiction vs autobiographie (pp.175-192) e Chronique de l'autofiction (pp. 193-212), fanno parte di quella che può essere considerata la seconda sezione del volume poiché entrambi completano Autofiction: une aventure du langage, testo di Gasparini del 2008: i contributi analizzano il termine di autofiction aprendo un dibattito sulla sua genesi ed evoluzione, passando in rassegna opere e scrittori che ne 
hanno determinato il riconoscimento letterario. L'A. parte dall'assunto che, per comprendere l'evoluzione di questa pratica ambigua, è necessario interrogarsi sulle origini del fenomeno, il quale si definisce proprio attraverso un'opposizione al genere stesso da cui deriva e con cui rischia di essere confuso - l'autobiografia.

4 Gli ultimi tre articoli si ricollegano invece al volume del 2013 La tentation autobiographique de l'Antiquité à la Renaissance, che mette in evidenza le differenze e variazioni tra le scritture dell'io secondo un'analisi diacronica e diatopica. Il primo, $L a$ tentation autofictionnelle de l'Antiquité à la Renaissance (pp. 213-242), propone uno studio delle strategie ambigue in prima persona sviluppate nei secoli che precedono il Seicento, testimoniando l'ibridazione dei generi e delle forme nelle antiche società, dall'antica Grecia all'antica Roma al Giappone. Pragmatique de la préface en régime autobiographique (pp. 243-260) presenta uno studio comparativo delle prefazioni ai testi autobiografici: un «tour d'horizon» da Montaigne a Senancour, Constant e Duras, per dimostrare come la Préface, la cui funzione è quella di precisare il contratto di lettura, giustificare, presentare liminarmente il testo, si sia via via indebolita lasciando spazio a un semplice titolo, sottotitolo o alle dichiarazioni dell'autore in quarta di copertina. Chiude il volume Vers une histoire des écritures du moi (pp. 261-270), una riflessione di genere sulle scritture dell'io e sul cammino intrapreso negli ultimi anni, in cui l'A. si sofferma in particolare sulle recenti evoluzioni delle équipe di ricerca e dei centri di studio. Con questo volume Gasparini non intende proporre nulla di nuovo su un tema già troppo discusso, ma completare e illustrare in modo puntuale e chiaro saggi teorici e testi relativi alle scritture in prima persona da lui pubblicati in precedenza. 